\title{
A ReaxFF Force Field for 2D-WS 2 and Its Interaction with Sapphire
}

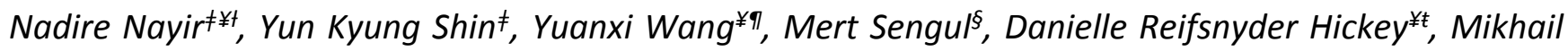 \\ Chubarov ${ }^{\ddagger}$, Tanushree H. Choudhury ${ }^{\ddagger}$, Nasim Alem ${ }^{\ddagger}$, Joan Redwing ${ }^{\ddagger}$, Vincent H. Crespi ${ }^{\ddagger}$, Adri C.T. van \\ Duin $\neq \#^{*}$
}

${ }^{\prime}$ Department of Mechanical Engineering, The Pennsylvania State University, University Park, Pennsylvania 16802, United States

¥2-Dimensional Crystal Consortium (2DCC) Materials Research Institute, The Pennsylvania State University, University Park, Pennsylvania 16802, United States

${ }^{t}$ Department of Physics, Karamanoglu Mehmetbey University, Karaman 70000, Turkey

" Department of Physics, The Pennsylvania State University, University Park, Pennsylvania 16802, United States

$\S$ Department of Pharmaceutical Sciences, University of Maryland Baltimore, Baltimore, MD, USA

${ }^{\ddagger}$ Department of Materials Science and Engineering, The Pennsylvania State University, University Park, Pennsylvania 16802, United States

\section{Theoretical Methods}

We developed a ReaxFF reactive force field parameter set for the W/S/Al/O/H interactions to study the energetics and the reaction mechanisms in the $2 \mathrm{D}-\mathrm{WS}_{2}$ on sapphire. The force field parameters were optimized against a Quantum-mechanics (QM) data which contains both non-periodic and condensed phase calculations.

DFT calculations: To prepare a QM-based training set, DFT calculations were performed using plane-wave basis first-principles code VASP $^{1}$ with projector augmented wave potential $\left.\right|^{2,3}$ and Perdew-Burke-Ernzerhof exchange-correlation potential ${ }^{4,5}$. For the $\mathrm{W} / \mathrm{S} / \mathrm{H}$ interactions, the same DFT settings as the works done by Ostadhussein et al $^{6}$., and Nayir et al. ${ }^{7,8}$, were applied to periodic and non-periodic calculations. For the $\mathrm{Al} / \mathrm{O} / \mathrm{S}$ interactions, calculations included spin polarization to properly describe the magnetic structure of alloy systems. During the lattice optimization of bulk $\mathrm{Al}_{2} \mathrm{O}_{3-\mathrm{x}} \mathrm{S}_{\mathrm{x}}$ crystals, an energy cut-off of 500 eV was applied with a grid spacing of $0.20 \AA^{-1}$ to $0.25 \AA^{-1}$ between k-points in reciprocal space using the tetrahedron method with Blöchl corrections. All calculations were performed with an electronic threshold of $10^{-4} \mathrm{eV}$. For all other calculations, we applied an energy cut-off of $400 \mathrm{eV}$ with a grid spacing varying from $0.25 \AA^{-1}$ to $0.35 \AA^{-1}$. For slab systems, $20 \AA$ of vacuum separated the surfaces of adjacent slabs. Reciprocal space integration was performed over the points generated by the Monkhorst-Pack scheme. The energy cut-off employed here is based on the recommended value for sulfur. Transition state calculations for sulfur diffusion on an Al surface were performed with the nudged elastic band method with climbing-images $(\mathrm{Cl}-\mathrm{NEB})^{9,10}$, relaxing the trajectory until the net residual force on each image is less than $0.01 \mathrm{eV} / \AA . A$. The minimum-energy reaction pathway was found by linearly interpolating five images between the known initial and final states as an initial guess, and then minimizing the energy of this string of images.

Force Field Fitting Procedure: The W/S/H/Al/O QM dataset includes the formation energies of monolayer $1 \mathrm{~T}-\mathrm{W} \mathrm{S}_{2}$ and mono- and bilayer $2 \mathrm{H}$ phases (Figure 1 ) to properly account for interlayer interactions within the van der Waals layered WS2 structure. It is noteworthy that this force field is expected to provide a decent description for the bulk form, as well, since the interlayer interactions in $\mathrm{WS}_{2}$ (and other TMD materials) are governed by weak van der Waals forces, one is already accounted during the current force field training by including the bilayer structures. Additionally, the formation energies of various point defects in 2D$\mathrm{WS}_{2}$ (Figure Error! Reference source not found.) and $\mathrm{WS}_{2}$ zigzag edges with varying S-coverages (Figure S1, S2) were included as well as $\mathrm{Al}_{2} \mathrm{O}_{3-\mathrm{x}} \mathrm{S}_{\mathrm{x}}(\mathrm{x}=0-3)$ crystals with varying sulfur concentrations and interstitial sulfur atoms in an fcc-Al crystal (Figure S3). We

\footnotetext{
*Corresponding author - *Email: acv13@psu.edu
} 
also added the adsorption energies of a sulfur atom on various sites of $\mathrm{Al}(100)$ and (111) surfaces and the migration path of a sulfur atom on an $\mathrm{Al}(111)$ surface in order to describe surface segregation and diffusion (Figures S4-S6 and Tables S1, S2). The equations of state of $W_{2}$ monolayer with uniform in-plane strain and bilayer strained uniaxially along the c direction (Figure 1), and $\mathrm{PG}_{1}$ and corundum- $\mathrm{Al}_{2} \mathrm{~S}_{3}$ (Figure S3) deformed isotropically were investigated to ensure that material properties are described properly with our force field under a range of mechanical conditions. The non-periodic DFT calculations obtained the energy profiles for the distortion of the $\mathrm{S}=\mathrm{W}-\mathrm{SH}, \mathrm{HS}-\mathrm{W}-\mathrm{SH}$ and $\mathrm{S}=\mathrm{W}=\mathrm{S}$ angles in $\mathrm{W}(\mathrm{SH})_{2} \mathrm{~S}_{2}$; each angle was scanned from 60 to $180^{\circ}$ (Figure S7). The energy profiles of the $\mathrm{W}-\mathrm{SH}$ and $\mathrm{W}=\mathrm{S}$ bonds in a $\mathrm{W}(\mathrm{SH})_{2} \mathrm{~S}_{2}$ molecule were also included in the training set (Figure S8). To fit the force field parameters, we began with the S (W) atom, the S-S (W-W) bond and off-diagonal, and the S-S$\mathrm{S}$ valence angle parameters from Ref. 6 (7), respectively. The Al-atom, Al-Al and Al-O bond, Al-O off-diagonal and Al-O-related valence angle parameters were taken from Ref. 11. Then, we optimized the following potential parameters against the QM data set: $\mathrm{W}-\mathrm{S}, \mathrm{Al} / \mathrm{S}$ and $\mathrm{Al}-\mathrm{S}$ bond and off-diagonal parameters, W-S- and Al-S-related and O-Al-S valence angle parameters. Additionally, parameters describing the $\mathrm{W}-\mathrm{Al}-\mathrm{S}$ and $\mathrm{Al}-\mathrm{W}-\mathrm{S}$ angles were straight copied from that of the $\mathrm{W}-\mathrm{W}-\mathrm{S}$ angle. For the $\mathrm{W}-\mathrm{S}-\mathrm{Al}$ angle, we used equilibrium angle and force constant parameters close to the average values of the Al-S-Al and $\mathrm{W}-\mathrm{S}-\mathrm{W}$ angles. It is noteworthy that our current training set is not targeting the W/Al/S and Al/W/S specifically. As such, to avoid underfitting the ReaxFF parameters, it is common practice to copy these angle parameters from a set of related and well parameterized parameters and then keep them fixed through the parameterization. The W/W/S angle parameters are chemically the closest match to the $\mathrm{W} / \mathrm{Al} / \mathrm{S}$ and $\mathrm{Al} / \mathrm{W} / \mathrm{S}$ parameters - and they have fairly significant values, indicating that metal-metal-sulfur angles can not be ignored for these materials.

ReaxFF/CI-NEB and DFT/CI-NEB calculations: The ReaxFF based Cl-NEB calculations (Error! Reference source not found. andError! Reference source not found.) were conducted via ADF $12,13 /$ ReaxFF ${ }^{14}$ software. We first mapped out an approximate pathway including five replicas between the optimized reactant and product. Then a force-based optimizer ("FIRE" 15 in all ReaxFF/Cl-NEB calculations, and "Quick-min"10 in DFT for the $1 \mathrm{~T} \rightarrow 2 \mathrm{H}$ phase transition) relaxed the trajectory through Cl-NEB sampling until the net force acting on each image reached $0.05 \mathrm{eV} / \AA ̊ \AA$ at both the DFT and ReaxFF levels by applying "fix atom" constraint only to the $\mathrm{W}$ atomic positions within a climbing image scheme.

The simulation box dimensions adopted for the phase transition calculations are as follows:

Figure 2: A hexagonal supercell with the dimensions of $6.34 \times 3.17 \times 20 \AA^{3}$ and $6.38 \times 3.19 \times 20 \AA^{3}$, for $2 \mathrm{~h}$ and $1 T$ phases, respectively.

Figure 3: An orthogonal simulation box with the dimensions of $21.956 \times 19.015 \times 30.00 \AA^{3}$ for the Pristine and SV models, and $40.00 \times 19.743 \times 30.00 \AA^{3}$ for each edge model.

ReaxFF Molecular Dynamics Simulations: Geometrical optimization of each model presented in this work was conducted using the low temperature molecular dynamics method via ADF ${ }^{12,13} /$ ReaxFF $^{14}$ to locate their lowest energy configuration. Temperature was set to $0.1 \mathrm{~K}$ in an NVT ensemble using the Berendsen thermostat ${ }^{16}$ with a coupling constant of $100 \mathrm{fs}$. The velocity-Verlet algorithm ${ }^{17}$ is adopted to integrate Newton's equation of motion and the time step value is set to 0.25 fs to ensure energy conservation during the simulation. It is noteworthy that ReaxFF MD works typically utilize a shorter time step in simulations than non-reactive force fields, which is $0.10 \mathrm{fs}$ or $0.25 \mathrm{fs}$ since the bond orders and charges are updated at every time step which requires a smaller time step than the usual in order to ensure energy conservation. For example, Rajabpour et al ${ }^{18}$ show that a time step of $0.25 \mathrm{fs}$ or $0.1 \mathrm{fs}$ allows for high temperature thermal decomposition to occur efficiently and with good energy conservation in ReaxFF MD simulations. However, the potential energies at $300 \mathrm{~K}, 1000 \mathrm{~K}$, and $2800 \mathrm{~K}$ with a time step of $1.00 \mathrm{fs}$ all deviate from the curves computed by the time steps of $0.10 \mathrm{fs}$ and $0.25 \mathrm{fs}$. Especially, in Figure S11 (e) and (f) of Ref. 18, the numbers of hydrogen gas molecules at high temperatures, i.e., $1000 \mathrm{~K}$ and $2800 \mathrm{~K}$, indicate that there is a big discrepancy between the time step of $1.00 \mathrm{fs}$ and the other three-timesteps. In addition, even with a time step of $0.50 \mathrm{fs}$, the potential energy and the number of hydrogen gas molecules at $2800 \mathrm{~K}$ are both slightly off the curves computed by the time steps of $0.10 \mathrm{fs}$ and $0.25 f s$.

In Figure 6, the optimized configuration of $\mathrm{WS}_{2}$ on Al-terminated c-sapphire (six double Al layers, separated by five $\mathrm{O}$ layers) was further subject to the equilibration at $300 \mathrm{~K}$ in an orthogonal simulation box with the dimensions of $101.21 \times 110.92 \times 50.00 \AA^{3}$ to explore the lattice reconstructions, and energetic of a slanted translational grain boundary observed in a $\mathrm{WS}_{2}$ thin film. The box dimensions for the rest of the calculations are as follows: an orthogonal simulation box with the dimensions of $40.00 \times 31.69 \times$ $30.00 \AA^{3}$ for Figures 4 and S10, $43.91 \times 44.3 \times 40 \AA^{3}$ for Figures 5 and S11 and $109.78 \times 126.76 \times 50.00 \AA^{3}$ for Figure 8, and a hexagonal simulation box with the dimensions of $19.01 \times 19.01 \times 19.26$ for Figure 7. 


\section{EXPERIMENTAL METHOD}

Synthesis of 2D-WS $\mathbf{2}$ film: WS $_{2}$ films were synthesized on $\mathbf{2}^{\prime \prime}$ epi-ready double side polished c-sapphire substrates in a horizontal, cold-wall MOCVD reactor ${ }^{19}$. The substrates were placed on an inductively coupled graphite susceptor for the deposition. Tungsten hexacarbonyl $\left(\mathrm{W}(\mathrm{CO})_{6}\right)$ and hydrogen sulfide $\left(\mathrm{H}_{2} \mathrm{~S}\right)$ were introduced separately for the growth and $\mathrm{H}_{2}$ was used as the carrier gas. A total gas flow rate of $4500 \mathrm{sccm}$ and pressure of 50 Torr were used. The films were deposited using a multi-step process with nucleation at $850^{\circ} \mathrm{C}$ and lateral growth at $1000^{\circ} \mathrm{C}$ which resulted in coalesced monolayer growth across the $2^{\prime \prime}$ wafer in $10 \mathrm{~min}$. $\mathrm{W}(\mathrm{CO})_{6}$ flow was modulated with $1.3 \times 10^{-4} \mathrm{sccm}$ during the nucleation and $6.4 \times 10^{-5} \mathrm{sccm}$ during the ripening process. $400 \mathrm{sccm}$ of $\mathrm{H}_{2} \mathrm{~S}$ was used for the whole process. Additional details are discussed in Ref. 20.

Transfer and characterization details of 2D-WS $\mathbf{W}_{\mathbf{2}}$ : A PMMA-assisted transfer method was used to detach the WS $_{2}$ monolayer film from the sapphire substrate and attach it to a Quantifoil TEM grid. Further details can be found in Chubarov et al. (Ref. 20). For annular dark field-scanning transmission electron microscopy (ADF-STEM) an FEI Titan3 G2 was employed to image at an accelerating voltage of $80 \mathrm{kV}$, with a probe convergence angle of $21.4 \mathrm{mrad}$ and a current of $60 \mathrm{pA}$.

\subsection{Periodic Calculations}

\subsubsection{Edge formation energies of $\mathrm{WS}_{2}$ Nanoribbons}

The DFT-based energies and structures for four representative nanoribbon models illustrated in Figure S1 were introduced in the training set, then the force field parameters were fit against the DFT data. To compute the excess edge energies of these models, a supercell with the dimensions of $0.63 \times 2.6 \times 0.32 \mathrm{~nm}^{3}$ was adopted with the periodic boundary applied along the $x$-direction. To avoid the spurious interactions between the replicas, a vacuum region with a height of $1.5 \mathrm{~nm}$ was inserted along the $y$ and $\mathrm{z}$ directions. After the relaxation the structures within ReaxFF and DFT, the formation energy per atom of the representative edge models in

Figure S1 was computed using Eq. S1.

$\nu=\left[E_{w s 2}-n_{w s 2} \mu_{w s 2}+n_{s} \mu_{s}\right] / N$

where $\mathrm{N}$ stands for the number of atoms of ribbon. $\mathrm{E}_{\mathrm{Ws} 2}$ is the total energy of each representative nanoribbon model and $\mu_{\mathrm{ws} 2}$ is the energy of a WS $\mathrm{W}_{2}$ unit in a perfect monolayer. $\mathrm{n}_{\mathrm{Ws} 2}$ is the number of the $\mathrm{WS}_{2}$ unit in each configuration. $\mu_{\mathrm{S}}$ is the energy of a $\mathrm{S}$ atom in a $\alpha$-S bulk, $n_{S}$ is the number of extra $\mathrm{S}$-atom saturating edges.

(a) $\mathrm{S}_{100} \mathrm{~S}_{100}$

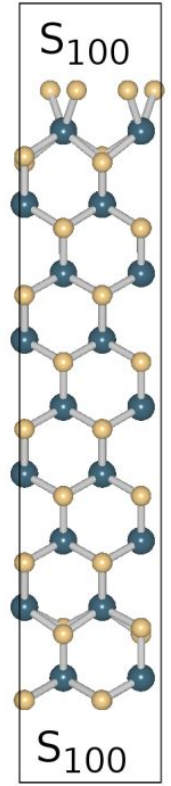

(b) $\mathrm{S}_{100} \mathrm{~S}_{50}$

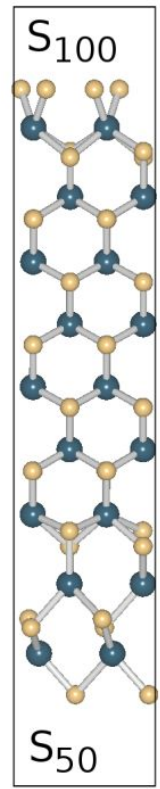

(c) $\mathrm{S}_{50} \mathrm{~S}_{50}$

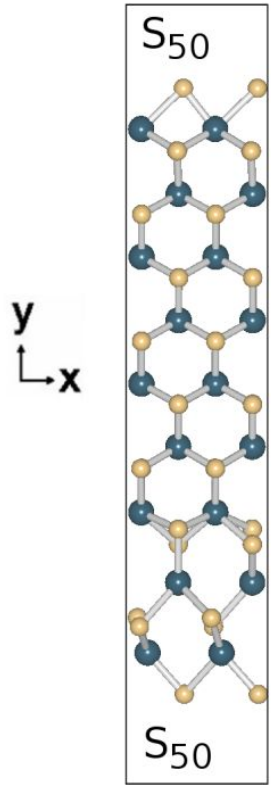

(d) $\mathrm{S}_{50} \mathrm{~S}_{100}$

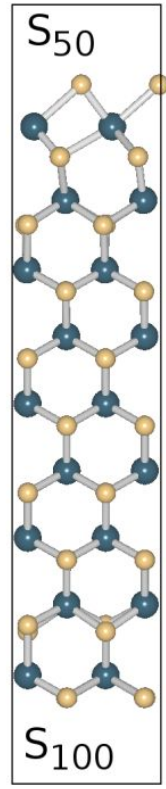


Figure S1 WS $\mathrm{W}_{2}$ nanoribbon models with zigzag edges covered by different S-concentrations. (a) $\mathrm{S}_{100} \mathrm{~S}_{100}$ model with both zigzag edges covered by $100 \% \mathrm{~S}$ atoms. (b) $\mathrm{S}_{100} \mathrm{~S}_{50}$ model with $100 \%$ and $50 \% \mathrm{~S}$-covered zigzag edges, (c) $\mathrm{S}_{50} \mathrm{~S}_{100}$ model with both edges covered by $50 \%$ S-atoms and (d) $\mathrm{S}_{50} \mathrm{~S}_{100}$ model with $50 \%$ and $100 \%$ S-covered zigzag edges.

Figure S2 presents the edge formation energies of four representative $\mathrm{WS}_{2}$ nanoribbon models where the ReaxFF energies of the models exhibits a similar trend with the DFT energies. The $\mathrm{S}_{100} \mathrm{~S}_{100}$ model is found as a most stable edge type in S-rich condition, followed by $S_{100} S_{50}$, then $S_{50} S_{100}$. The stability of the stoichiometric $S_{50} S_{50}$ remains same in all growth conditions, and determined as a least stable edge in the S-rich environment at both ReaxFF and DFT levels. Towards the W-rich conditions, the stability of $\mathrm{S}_{100} \mathrm{~S}_{100}, \mathrm{~S}_{100} \mathrm{~S}_{50}$ and $\mathrm{S}_{50} \mathrm{~S}_{100}$ models show a decreasing trend - among them, the $\mathrm{S}_{100} \mathrm{~S}_{50}$ edge become a most stable edge. Additionally, both ReaxFF and DFT results show that the edge energies of $S_{100} S_{50}$ and $S_{50} S_{50}$ models as well as $S_{50} S_{100}$ and $S_{100} S_{100}$ energies converges to each other towards the W-rich condition. Note that, in W-rich environment, ReaxFF predicts the $S_{100} S_{100}$ and $\mathrm{S}_{100} \mathrm{~S}_{50}$ models thermodynamically more stable than the DFT method while ReaxFF-based $S_{50} S_{100}$ and $S_{50} S_{50}$ exhibit the less thermodynamic stability than the ones at the DFT level.

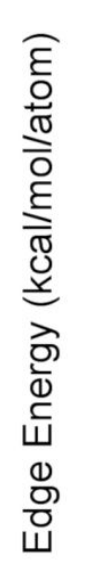

a

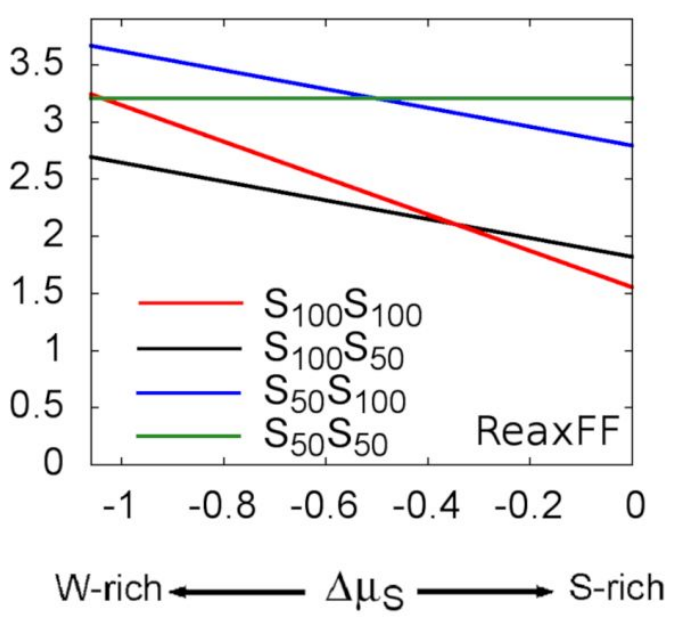

b

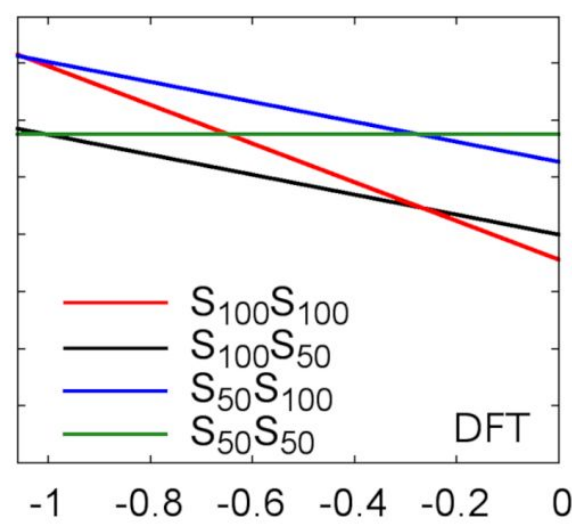

$\begin{array}{llllll}-1 & -0.8 & -0.6 & -0.4 & -0.2 & 0\end{array}$

Figure S2 (a) The ReaxFF and (b) DFT-based excess energies of the $\mathrm{WS}_{2}$ edges with different S-coverages in

Figure S1, as a function of $\Delta \mu_{\mathrm{S}}$ which is is the excess chemical potential which is the difference between $\mu_{\mathrm{S}}$ and $\mu_{\mathrm{s}}$ (bulk). Note that $\mu_{S(\text { bulk) }}$ is the energy per atom of bulk $\alpha-S$.

\subsection{2 $\mathbf{W S}_{\mathbf{2}}$ interaction with Sapphire}

$\alpha-\mathrm{Al}_{2} \mathrm{O}_{3}$ known as sapphire and belonging to the space group of $\mathrm{R3} c$, is one of the common oxides used in the TMD growth because of its hexagonal symmetry, good chemical and thermal stability at the elevated temperatures and commercial availability. The $\mathrm{Al} / \mathrm{O} / \mathrm{H}$ ReaxFF force field reported in earlier works ${ }^{1-3}$ is trained against an extensive DFT dataset including heats of formation and equations of state of different polymorphs of $\mathrm{Al}_{2} \mathrm{O}_{3}$ as well as the bond-angle energetics of $\mathrm{Al}(\mathrm{OH})_{3}$ and $(\mathrm{OH})_{2} \mathrm{Al}$ $\mathrm{Al}(\mathrm{OH})_{2}$ molecules and reaction energies of $\mathrm{Al} / \mathrm{O} / \mathrm{H}$ compounds. In this work we extended the ReaxFF force field to the $\mathrm{W} / \mathrm{S} / \mathrm{H} / \mathrm{Al} / \mathrm{O}$ interactions to describe the $\mathrm{WS}_{2}$ interactions with sapphire. 
Crystal stability of $\mathrm{Al}_{2} \mathrm{~S}_{3}$ and S-impurity in Sapphire: In order to obtain the energy-volume relations of $\mathrm{PG}_{1}-\mathrm{Al}_{2} \mathrm{~S}_{3}$ and the corundum phase, the volume of the unit cell was compressed and expanded isotropically. As shown in Figure $S 3 a-d, P 6_{1}-A I_{2} S_{3}$ is the ground state with the formation energy of $-153.5 \mathrm{kcal} / \mathrm{mol}$, while the corundum phase (R-3c) is $5.1 \mathrm{kcal} / \mathrm{mol}$ less stable than $\mathrm{P}_{1}-\mathrm{Al}_{2} \mathrm{~S}_{3}$ in ReaxFF, which is in a good agreement with the DFT data. Under high pressure, the corundum phase becomes thermodynamically more favorable, probably inducing structural phase transition. The bulk modulus $(B)$ of $P 6_{1}-A_{2} S_{3}$ calculated from the equation of state is $30.8 \mathrm{GPa}$ in ReaxFF, which is in good agreement with DFT (34.8 GPa). However, it should be cautious to apply this force field to the study of mechanical properties of the corundum phase since its bulk modulus (70.0 GPa) is underestimated by $20 \%$ ( $88.3 \mathrm{GPa}$ in DFT).

(a) $\mathrm{P}_{1}$

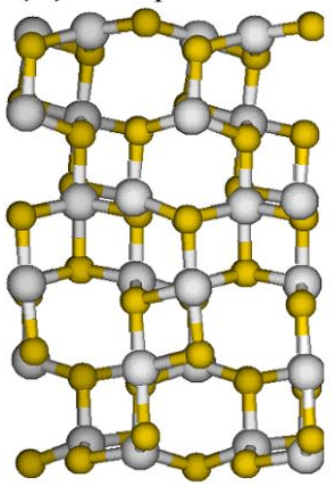

(b) corundum

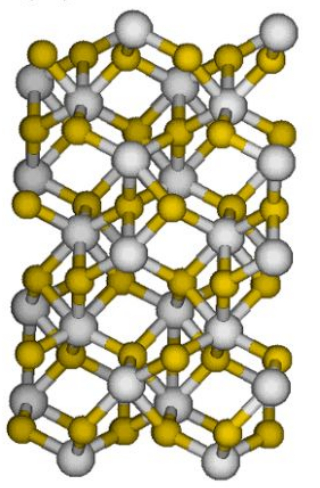

(c)

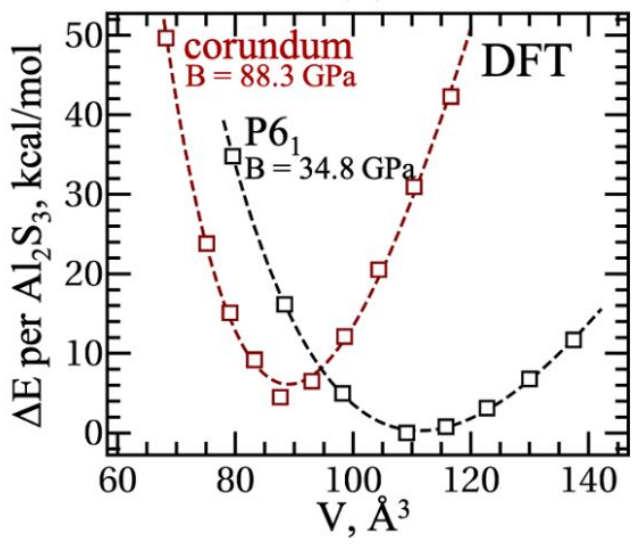

(d)

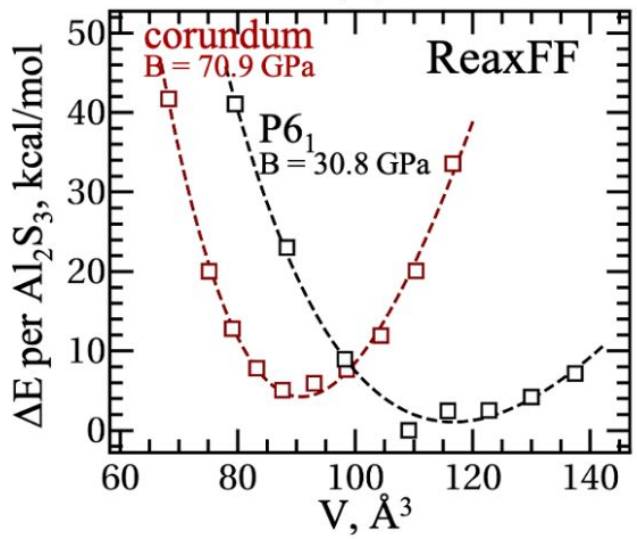

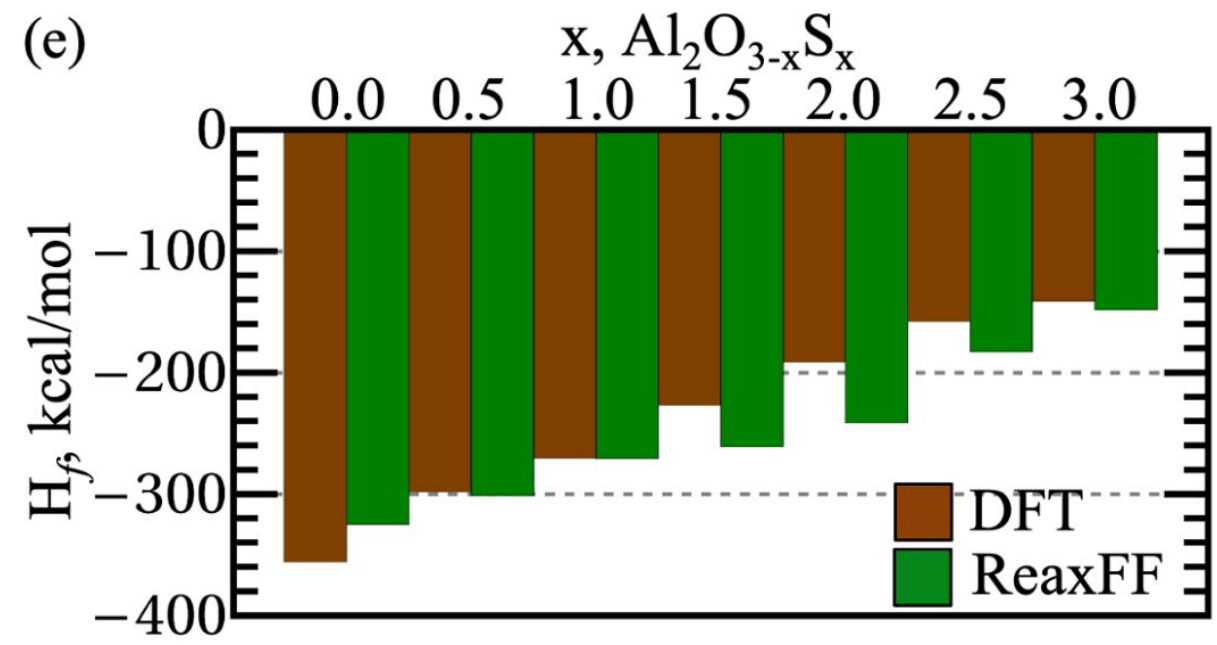

Figure S3: $(\mathrm{a}, \mathrm{b})$ Atomic configurations and (c, d) equations of state of $\mathrm{P}_{1}$ - and corundum- $\mathrm{Al} \mathrm{S}_{3}$, respectively. (e) Heat of formation of $\mathrm{Al}_{2} \mathrm{O}_{3-\mathrm{x}} \mathrm{S}_{\mathrm{x}}(\mathrm{x}=0-3)$ at $0 \mathrm{~K}$. $\mathrm{Al}_{2} \mathrm{O}_{3-\mathrm{x}} \mathrm{S}_{\mathrm{x}}$ with low sulfur concentration is more stable than that with high sulfur concentration.

Sulfur, as an impurity on metallic oxide plays a crucial role in reducing the stability of the protective oxide layer and degrading the mechanical properties of the material. To describe the thermodynamic stability of $\mathrm{Al}_{2} \mathrm{O}_{3}$ in the presence of sulfur, corundum$\mathrm{Al}_{2} \mathrm{O}_{3-\mathrm{x}} \mathrm{S}_{\mathrm{x}}(\mathrm{x}=0-3)$ structures with various concentrations of sulfur were added to the training set, and O-Al-S valence angle parameters were optimized. Different compositions of $\mathrm{Al}_{2} \mathrm{O}_{3-\mathrm{x}} \mathrm{S}_{\mathrm{x}}$ were obtained by replacing oxygen atoms with sulfur atoms. As shown in Figure S3e, the structure replaced by sulfur is energetically less favorable than $\mathrm{Al}_{2} \mathrm{O}_{3}$.

S adsorption and diffusion on $\mathbf{A l}(\mathbf{1 0 0})$ and $\mathbf{A l}(\mathbf{1 1 1})$ : Table S1 shows the comparison of ReaxFF and DFT data in various surface adsorption sites of sulfur on $\mathrm{Al}(100)$ and $\mathrm{Al}(111)$ at the coverage of $0.25 \mathrm{ML}$, e.g., top, bridge, hollow, fcc, hcp and octahedral interstitial site in subsurface (Figures S4-S5). The adsorption energy was calculated by: $E_{a d}(S)=[E(A l$ slab + Sulfur) $-E(A l$ slab) - 
$n(S)$ ] $E(a-S)] / n(S)$. Here $n(S)$ is the number of sulfurs on the Al surface. The hollow site on $\mathrm{Al}(100)$ surface and fcc site on $\mathrm{Al}(111)$ are the most stable sites. At higher sulfur coverages, the adsorption energy per sulfur decreases, due to the electrostatic repulsion between sulfur atoms, but is still negative on both (111) and (100) surfaces. When the Al surface is completely covered by one layer of sulfur $(1.0 \mathrm{ML})$, the adsorption energy is $-17.2 \mathrm{kcal} / \mathrm{mol}$ on (111) surface and $-21.7 \mathrm{kcal} / \mathrm{mol}$ on (100) surface in

ReaxFF. The adsorption energy at various coverages in ReaxFF and DFT is summarized in Table S2.
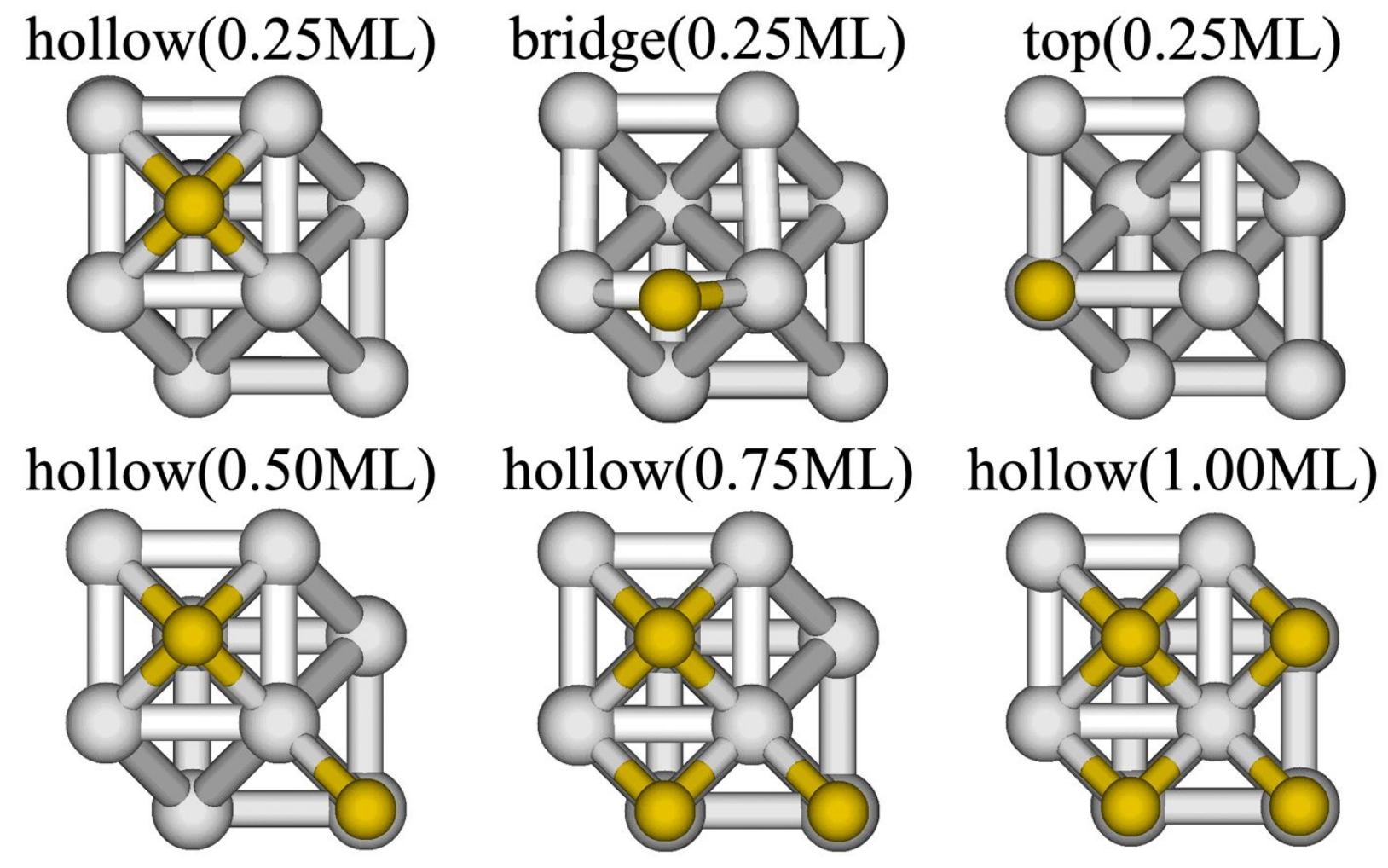

Figure S4: Sulfur binding sites on Al(100) surface.
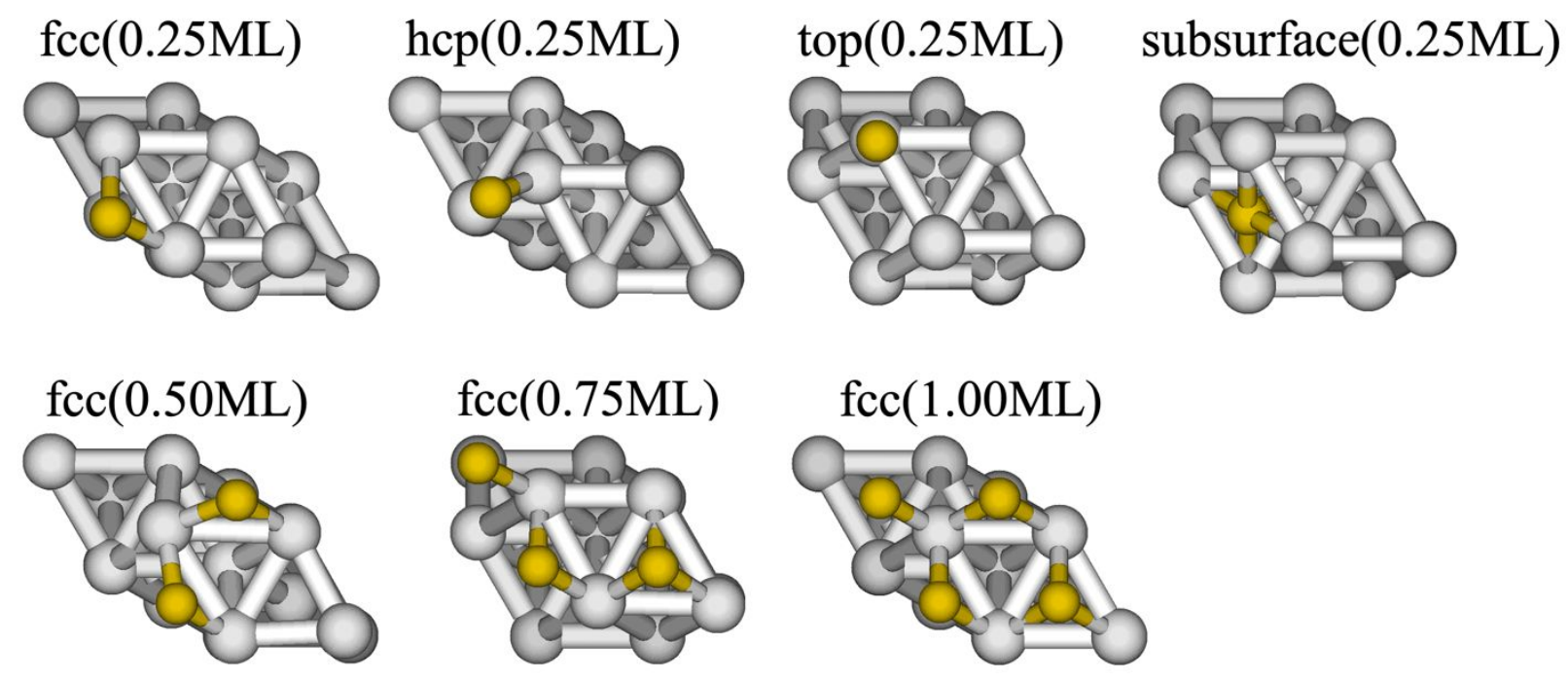

Figure S5: Sulfur binding sites on Al(111) surface. 
Table S1 Adsorption energy (in $\mathrm{kcal} / \mathrm{mol}$ ) of sulfur on various sites of $\mathrm{Al}(100)$ and (111) surfaces.

\begin{tabular}{lll}
\hline Adsorption site on $\mathrm{Al}(100)$ & DFT & ReaxFF \\
\hline Hollow & -39.79 & -43.50 \\
\hline Bridge & -36.92 & -42.85 \\
\hline Top & -18.90 & -19.20 \\
\hline Adsorption site on Al(111) & & \\
\hline Fcc & -52.15 & -35.76 \\
\hline Hcp & -46.35 & -26.82 \\
\hline Top & -15.80 & -16.97 \\
\hline Subsurface & 11.23 & 16.44 \\
\hline
\end{tabular}

Table S2 Adsorption energy of sulfur at various coverages on hollow and fcc site of Al surface.

\begin{tabular}{lll}
\hline Coverage on $\mathrm{Al}(100)$ & DFT & ReaxFF \\
\hline 0.25 & -39.79 & -43.50 \\
\hline 0.50 & -39.72 & -32.26 \\
\hline 0.75 & -25.79 & -26.73 \\
\hline 1.00 & -18.14 & -21.68 \\
\hline & & \\
\hline Coverage on $\mathrm{Al}(111)$ & & -35.76 \\
\hline 0.25 & -52.15 & -28.46 \\
\hline 0.50 & -33.95 & -22.36 \\
\hline 0.75 & -20.14 & -17.16 \\
\hline 1.00 & -12.48 & \\
\hline
\end{tabular}

The diffusion of sulfur on the (111) surface was studied at the low coverage. Figure S6a shows the energetics of diffusion from hcp to fcc site and the interstitial site of subsurface to fcc site. Sulfur on the interstitial site is thermodynamically unstable as calculated in Table S1. Placing a S atom to subsurface (octahedral site) introduces strains into the local structure. Because the inplane lattice dimension is fixed, the surface-to-subsurface interlayer spacing increases to release strains. This causes breaking of Al-Al bonds between the layers, which requires prohibitively high energy. In particular, the diffusion from surface to subsurface needs to overcome an energy barrier of $>90 \mathrm{kcal} / \mathrm{mol}$, the diffusion of sulfur to subsurface is neither thermodynamically nor kinetically favorable. On the other hand, sulfur diffusion via surface sites is likely to occur, although the energy barrier for hcp-to$\mathrm{fcc}$ diffusion is slightly overestimated in ReaxFF (6.3 kcal/mol), compared to $4.3 \mathrm{kcal} / \mathrm{mol}$ in DFT.
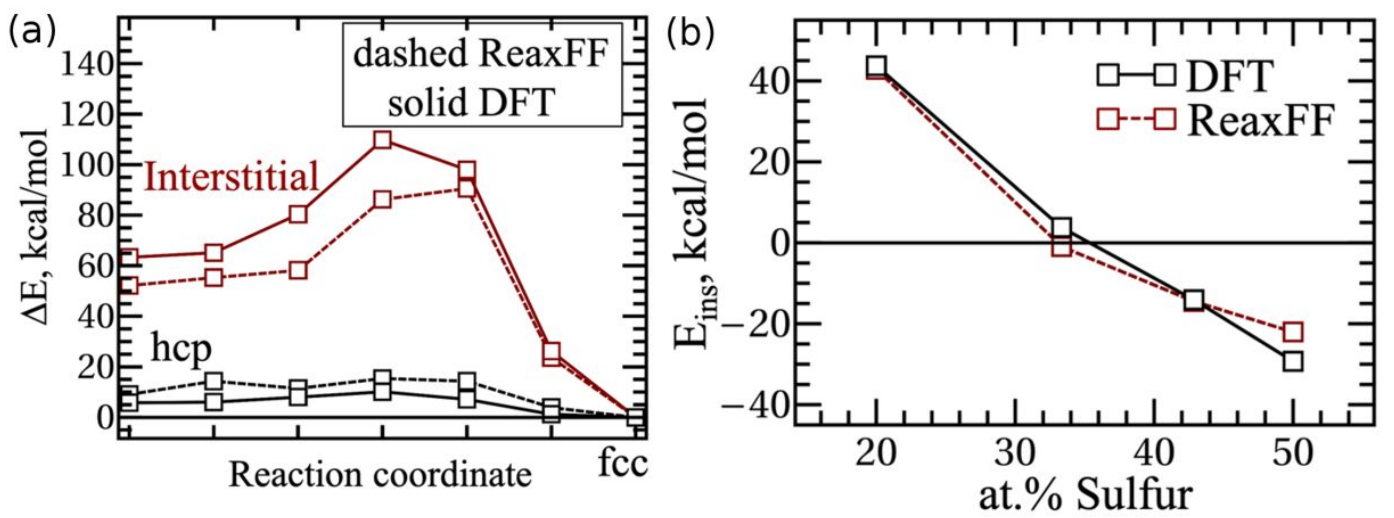
Figure S6: (a) Diffusion path of sulfur from hcp to fcc site and the interstitial to fcc site on $\mathrm{Al}(111)$ surface and (b) Insertion energy of sulfur into fcc $\mathrm{Al}$ at sulfur concentrations varying from 20 to 50 at.\%.

S defect on bulk Al: Since it is essential to understand sulfur segregation-induced transition of material properties, e.g., embrittlement and resistance to oxidation, we also investigated the thermodynamic behavior of S-doped Al at 20-50at.\% sulfur. We first inserted one sulfur atom into the interstitial at the octahedral site of 4 atom-fcc Al and sequentially occupied neighboring interstitial sites with sulfur atoms. As sulfur is inserted into fcc Al, the lattice parameter increases - from $3.981 \AA$ in fcc Al to $4.685 \AA$ (1S, 20at.\%), $4.781 \AA$ (2S, 33at.\%), $5.133 \AA$ (3S, 43at.\%) and $5.181 \AA$ (4S, 50at.\%). In particular, the first sulfur added to the lattice induces the lattice expansion of about $18 \%$, causing a longer Al-Al bond length and significant instability of the lattice. The instability caused by lattice expansion is recovered by occupying the space with additional $\mathrm{S}$ atoms and forming S-Al bonds. At 50at.\%, rock-salt AlS crystal is formed with the formation energy of $-22.0 \mathrm{kcal} / \mathrm{mol}$ per formula unit, which is less stable than $\mathrm{Al}_{2} \mathrm{~S}_{3}$. When the insertion energy is calculated by: $E_{\text {ins }}=[E(A l+S)-E(f c c A l)-n(S)$ ? $E(a-S)] / n(S)$, the DFT results predict positive insertion energy below 33at.\% of sulfur concentration (2S) but negative insertion energy at higher sulfur concentration. As shown in Figure S6b, the results in ReaxFF are in line with the DFT values.

1.2. W/S/H non-periodic calculations: As shown in Figure S7, the new ReaxFF predicts the same values for the equilibrium angles of $\mathrm{S}=\mathrm{W}=\mathrm{S}, \mathrm{HS}-\mathrm{W}-\mathrm{SH}$ and $\mathrm{S}=\mathrm{W}-\mathrm{SH}$ which are $115^{\circ}, 110^{\circ}$ and $105^{\circ}$, respectively, with DFT; however, it underestimates the QM data at shorter distances than the equilibrium angles (Figure S7-b,c). Figure S8 depicts the ReaxFF and DFT energy profiles of the single and double bonds between the $\mathrm{W}$ and $\mathrm{S}$ atoms. The spin state of the molecule in question differ in the well and tail regions of the bond dissociation curves, the ground state exhibits singlet state in the potential well, but changes to the triplet state at large separations. For the accurate description of this spin-flip behavior of the ground state depending on the separation distance between two atoms, each bond type was computed at the singlet and triplet state at the DFT level. The ReaxFF formulation does not describe the multi-spin behavior of the molecule changing depending on the separation distance, but it reproduces the energy associated with the lowest energy spin state. It can be seen in Figure S8 that ReaxFF fits well near the minimum points of the curves; the ReaxFF energy curves show a similar trend with the DFT ones. Additionally, ReaxFF predicts correctly the equilibrium values of $\mathrm{W}-\mathrm{S}$ and $\mathrm{W}=\mathrm{S}$ bonds to be 2.3 and $2.1 \AA$, respectively.

(a)

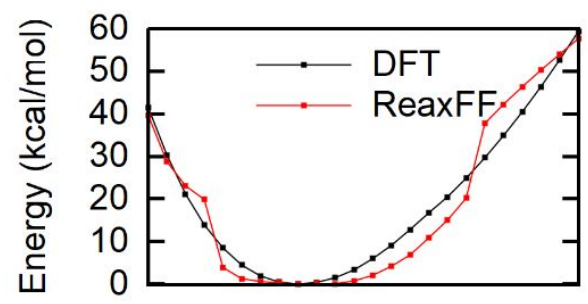

$\mathrm{S}=\mathrm{W}-\mathrm{SH}$ angle (deg) (b)

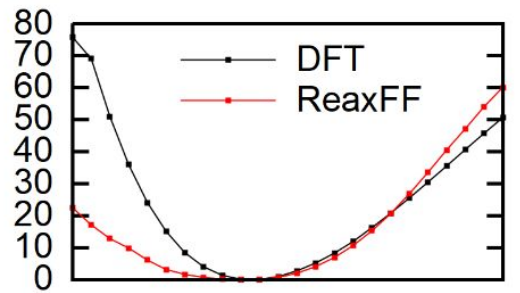

$\mathrm{S}=\mathrm{W}=\mathrm{S}$ angle (deg) (c)

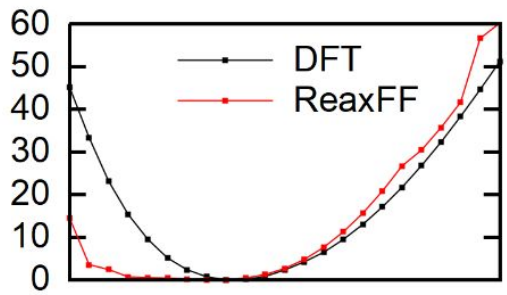

HS-W-SH angle (deg)

Figure S7: Bond-angle energetics of various angles in $\mathrm{W}(\mathrm{SH})_{2} \mathrm{~S}_{2}$ at the ReaxFF and DFT levels. (a) $\mathrm{S}=\mathrm{W}-\mathrm{SH},(\mathrm{b}) \mathrm{S}=\mathrm{W}=\mathrm{S}$ and (b) $\mathrm{HS}$ W-SH angles. 
(a)

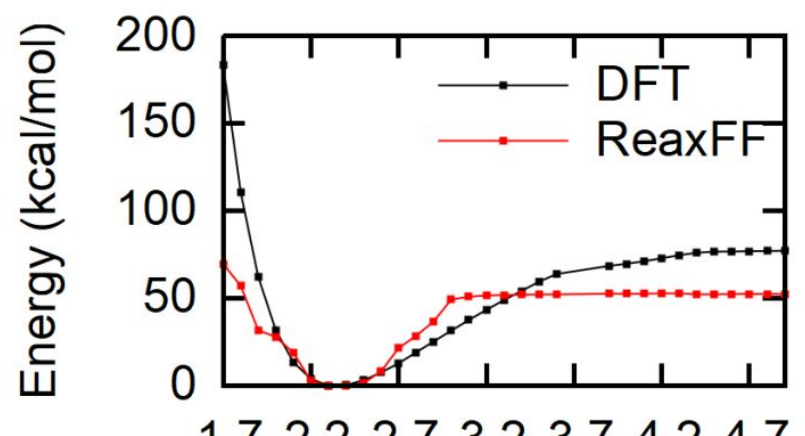

W-SH bond distance $(\AA)$ (b)

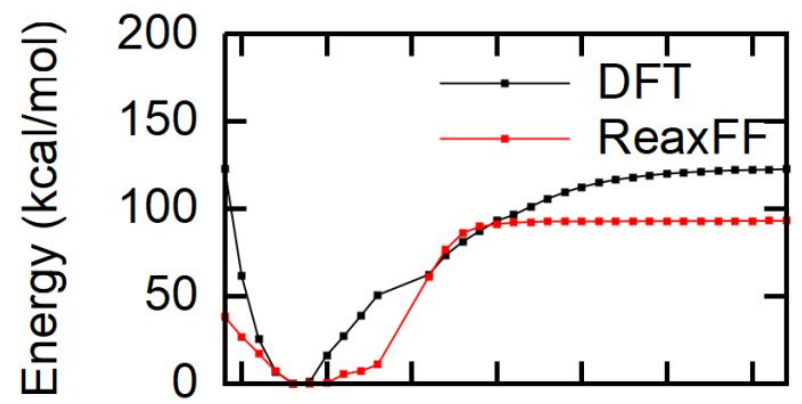

$\begin{array}{lllllll}1.8 & 2.32 .83 .3 & 3.8 & 4.3 & 4.8\end{array}$

$\mathrm{W}=\mathrm{S}$ bond distance $(\AA)$

Figure S8: Energy profiles of the (a) HS-W and (b) $\mathrm{S}=\mathrm{W}$ bond distortions in $\mathrm{W}(\mathrm{SH})_{2} \mathrm{~S}_{2}$ molecule at the ReaxFF and DFT levels.

\section{Force Field Applications to 2D-WS}

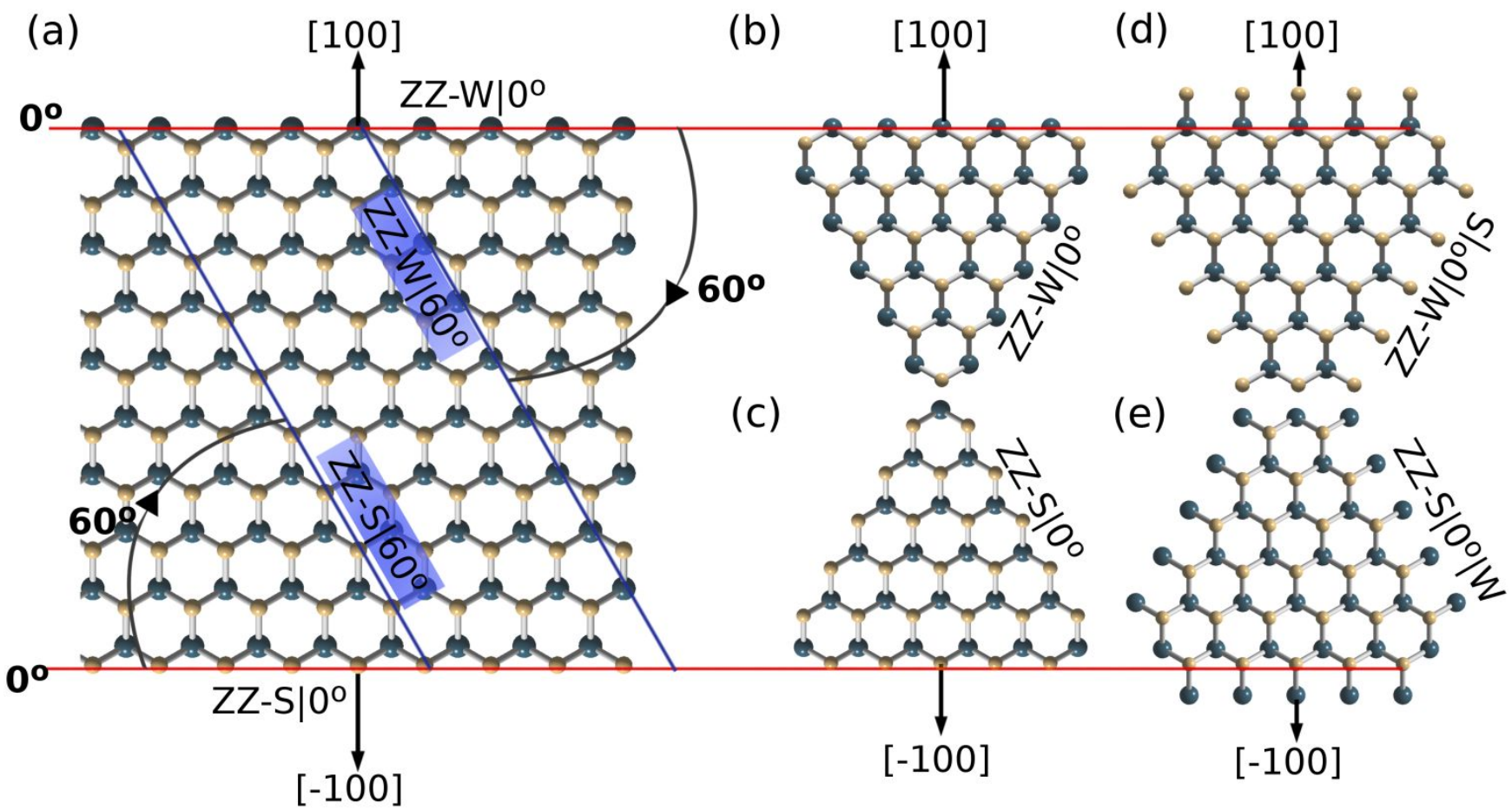

Figure S9 Metallic and chalcogen zigzag edges of 2D-WS . (a) A WS 2 nanoribbon model with metal (ZZ-W) and chalcogen (ZZ-S) terminated zigzag edges. ZZ-W $\mid 0^{\circ}$ and $\mathrm{ZZ}-\mathrm{S} \mid 0^{\circ}$ are aligned at $0^{\circ}$ but with opposite growth directions of [100] and [-100], respectively. ZZ-S $60^{\circ}\left(\mathrm{ZZ}-\mathrm{W} \mid 60^{\circ}\right)$ is obtained by the rotation of ZZ-S $\mid 0^{\circ}\left(\mathrm{ZZ}-\mathrm{W} \mid 0^{\circ}\right)$ by $60^{\circ}(\mathrm{b}, \mathrm{c})$ Triangle WS2 domain with ZZ$\mathrm{W}\left|0^{\circ} \mathrm{ZZ}-\mathrm{S}\right| 0^{\circ}$ edges, respectively. (d) ZZ-W $\left|0^{\circ}\right| \mathrm{S}$ is a metallic edge but saturated by S-atoms. (e) ZZ-S $\left|0^{\circ}\right| \mathrm{W}$ is a chalcogen edge but saturated by $W$-atoms. During the growth, the edge of a growth front interchangeably switches between the metallic edges (ZZ-W $\mid 0^{\circ}$ to $\mathrm{ZZ}-\mathrm{W}\left|0^{\circ}\right| \mathrm{S}$ or vice versa) or chalcogen edges (ZZ-S $\mid 0^{\circ}$ to ZZ-S $\left|0^{\circ}\right| \mathrm{W}$ or vice versa) by addition of extra one atomic row to the edge. 

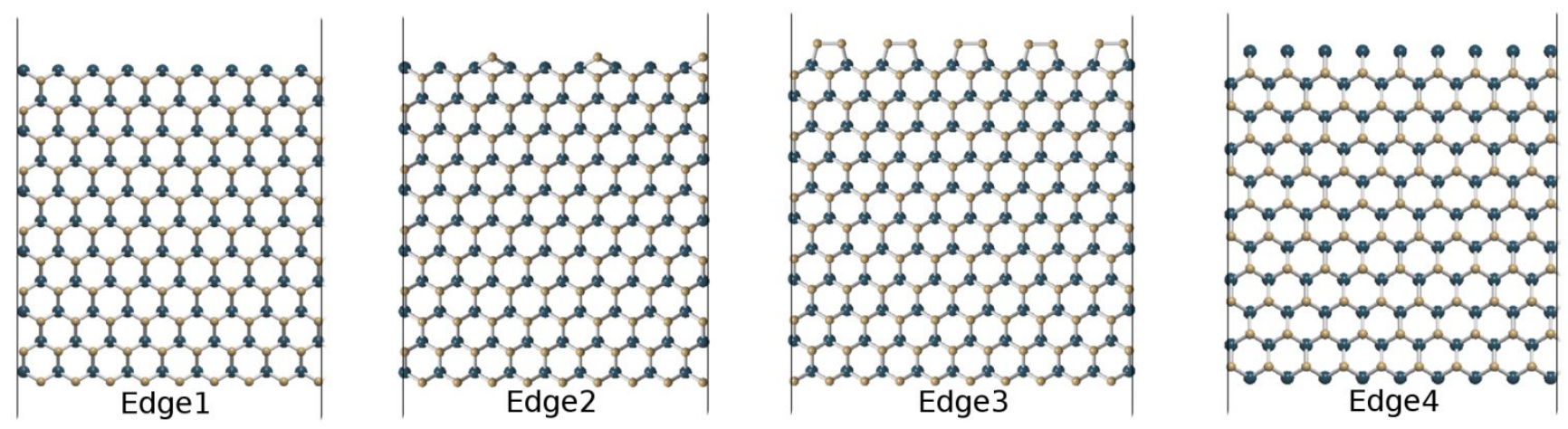

Figure S10 Atomic models of the representative edge types. Both "Edge1" and Edge2 consist of ZZ-S|0 and ZZ-W|0 but ZZ$\mathrm{W} \mid 0$ ㅇ of "Edge2" is partially saturated by S atoms. "Edge3" is the fully-S terminated and consists of a reconstructed ZZ-W|0|S and ZZ-S|O․ Lastly, the fully W-terminated "Edge4" contains a ZZ-S $\mid 0$ O $\mid \mathrm{W}$ and ZZ-W|0․

Excess energies of the representative edge models in

Figure S10 was computed using Eq. S2.

$V=\left[E_{w s 2}-n_{w s 2} \mu_{w s 2}+n_{s} \mu_{s}+n_{w} \mu_{w}\right] / 2 L \quad(W i d t h=2.6 n m, L=0.63 n m)$

where $L$ and $W$ stand for the length and width of ribbon, respectively. $E_{W s 2}$ is the total energy of each representative nanoribbon model and $\mu_{\mathrm{ws} 2}$ is the energy of a WS $\mathrm{W}_{2}$ unit in a perfect monolayer. $\mathrm{n}_{\mathrm{Ws} 2}$ is the number of the $\mathrm{WS}_{2}$ unit in each configuration. $\mu_{\mathrm{S}}$ and $\mu_{\mathrm{W}}$ are the energies of a $S$ and $W$ atom in a $\alpha-S$ and $W$-bcc bulks, $n_{S}$ and $n_{W}$ are the number of extra $S$ and $W$ atoms saturating edges, respectively. 
(a)

(b)

(c)

(d)
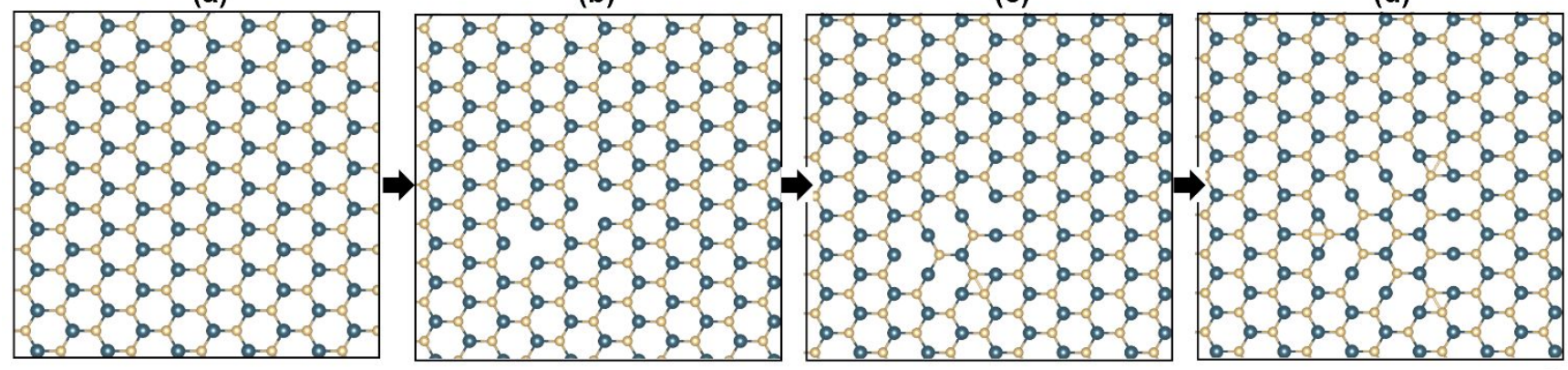

(h)

(g)

(f)

(e)

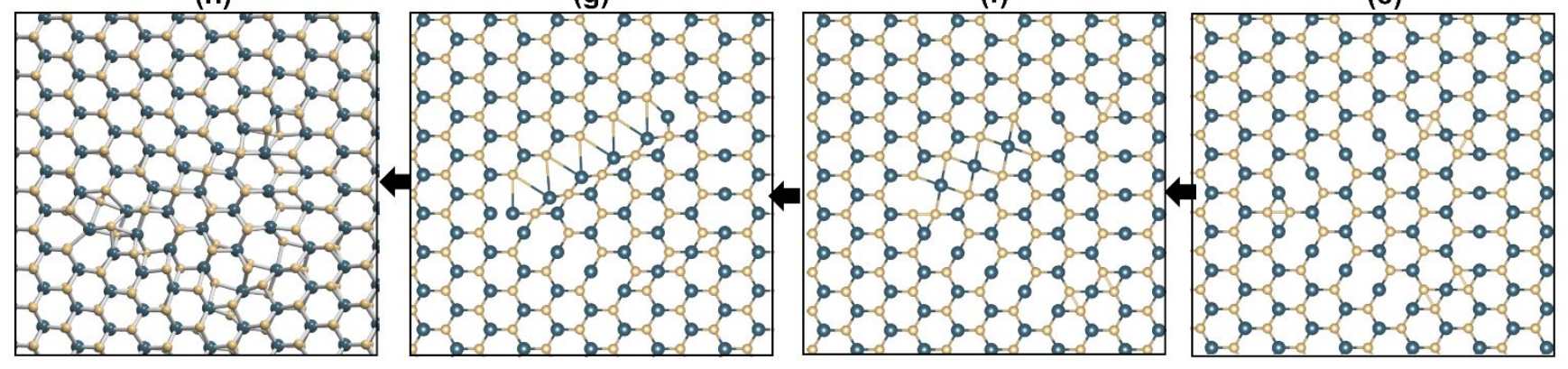

Figure S11: The atomistic modeling steps of the twin boundary shown in Fig. 5c, starting from the pristine structure. (a) A pristine $W_{2}$ (b) creating two adjacent divacancies, (c-e) the subsequent formation of three-fold rotational defects, (f) the translation of $\mathrm{W}$ atoms by a half of the W-W distance and (g) obtaining the initial configuration of the computed model presented in $\mathrm{h}$ and Figure 5c.

\section{References}

(1) Kresse, G.; Furthmüller, J. Efficient Iterative Schemes for Ab Initio Total-Energy Calculations Using a Plane-Wave Basis Set. Phys. Rev. B 1996, 54 (16), 11169-11186. https://doi.org/10.1103/PhysRevB.54.11169.

(2) Kresse, G.; Joubert, D. From Ultrasoft Pseudopotentials to the Projector Augmented-Wave Method. Phys. Rev. B 1999, 59 (3), 1758-1775. https://doi.org/10.1103/PhysRevB.59.1758.

(3) Blöchl, P. E. Projector Augmented-Wave Method. Phys. Rev. B 1994, 50 (24), 17953-17979. https://doi.org/10.1103/PhysRevB.50.17953.

(4) Perdew, J. P.; Burke, K.; Ernzerhof, M. Generalized Gradient Approximation Made Simple. Phys. Rev. Lett. 1996, 77 (18), 3865-3868. https://doi.org/10.1103/PhysRevLett.77.3865.

(5) Perdew, J. P.; Burke, K.; Ernzerhof, M. Generalized Gradient Approximation Made Simple [Phys. Rev. Lett. 77, 3865 (1996)]. Phys. Rev. Lett. 1997, 78 (7), 1396-1396. https://doi.org/10.1103/PhysRevLett.78.1396.

(6) Ostadhossein, A.; Rahnamoun, A.; Wang, Y.; Zhao, P.; Zhang, S.; Crespi, V. H.; van Duin, A. C. T. ReaxFF Reactive ForceField Study of Molybdenum Disulfide (MoS2). J. Phys. Chem. Lett. 2017, 8 (3), 631-640.

https://doi.org/10.1021/acs.jpclett.6b02902.

Nayir N., Wang Y., Shabnam S., Reifsnyder Hickey D., Mao L., Zhang X., Bachu S., Redwing J., Alem N., Crespi V. H., van Duin A. C. T, Modeling for Structural Engineering and Synthesis of Two Dimensional WSe2 Using a Newly Developed ReaxFF Reactive Force Field, J. Phys. Chem. C 2020, 124, 51, 28285-28297, https://pubs.acs.org/doi/abs/10.1021/acs.jpcc.0c09155

(8) Nayir N., Wang Y., Y. Ji, Choudhury T. H., Redwing J. M., Chen L.-Q., Crespi V. H., van Duin A. C. T., Theoretical Modeling of Edge-Controlled Growth Kinetics and Structural Engineering of 2D-MoSe $2, M S E B, 2021,271,115263$

(9) Henkelman, G.; Uberuaga, B. P.; Jónsson, H. A Climbing Image Nudged Elastic Band Method for Finding Saddle Points and Minimum Energy Paths. J. Chem. Phys. 2000, 113 (22), 9901-9904. https://doi.org/10.1063/1.1329672. 
(10) H. Jo'nsson; G. Mills; K. W. Jacobsen. Nudged Elastic Band Method for Finding Minimum Energy Paths of Transitions'. In Classical and Quantum Dynamics in Condensed Phase Simulations edited by B. J. Berne, G. Ciccotti, and D. F. Coker; World Scientific: Singapore, 1998; p 385.

(11) Hong, S.; van Duin, A. C. T. Molecular Dynamics Simulations of the Oxidation of Aluminum Nanoparticles Using the ReaxFF Reactive Force Field. J. Phys. Chem. C 2015, 119 (31), 17876-17886. https://doi.org/10.1021/acs.jpcc.5b04650.

(12) Amsterdam Modeling Suite Making Computational Chemistry Work For You https://www.scm.com/ (accessed Nov 10, 2020).

(13) Velde, G. te; Bickelhaupt, F. M.; Baerends, E. J.; Guerra, C. F.; Gisbergen, S. J. A. van; Snijders, J. G.; Ziegler, T. Chemistry with ADF. Journal of Computational Chemistry 2001, 22 (9), 931-967. https://doi.org/10.1002/jcc.1056.

(14) Plimpton, S. Fast Parallel Algorithms for Short-Range Molecular Dynamics. Journal of Computational Physics 1995,117 (1), 1-19. https://doi.org/10.1006/jcph.1995.1039.

(15) Bitzek, E.; Koskinen, P.; Gähler, F.; Moseler, M.; Gumbsch, P. Structural Relaxation Made Simple. Phys. Rev. Lett. 2006, 97 (17), 170201. https://doi.org/10.1103/PhysRevLett.97.170201.

(16) Berendsen, H. J. C.; Postma, J. P. M.; van Gunsteren, W. F.; DiNola, A.; Haak, J. R. Molecular Dynamics with Coupling to an External Bath. J. Chem. Phys. 1984, 81 (8), 3684-3690. https://doi.org/10.1063/1.448118.

(17) Verlet, L. Computer "Experiments" on Classical Fluids. I. Thermodynamical Properties of Lennard-Jones Molecules. Phys. Rev. 1967, 159 (1), 98-103. https://doi.org/10.1103/PhysRev.159.98.

(18) Rajabpour, S.; Mao, Q.; Nayir, N.; Robinson, J.; van Duin A. C. T. Development of a ReaxFF Reactive Force Field for Ga/C/H and In/C/H Interactions. J. Phys. Chem. C 2021, 125, 19, 10747-10758

(19) Xuan, Y.; Jain, A.; Zafar, S.; Lotfi, R.; Nayir, N.; Wang, Y.; Choudhury, T. H.; Wright, S.; Feraca, J.; Rosenbaum, L.; Redwing, J. M.; Crespi, V.; van Duin, A. C. T. Multi-Scale Modeling of Gas-Phase Reactions in Metal-Organic Chemical Vapor Deposition Growth of WSe2. Journal of Crystal Growth 2019, 527, 125247.

https://doi.org/10.1016/j.jcrysgro.2019.125247.

(20) Chubarov, M.; Choudhury, T. H.; Reifsnyder Hickey, D.; Bachu, S.; Zhang, T.; Sebastian, A.; Bansa, A.; Das, S.; Terrones, M.; Alem, N.; Redwing, J. M. Wafer-Scale Epitaxial Growth of Single Orientation WS2 Monolayers on Sapphire.

arXiv:2006.10952 [cond-mat] 2020. 